\title{
On the Bohr-Sommerfeld formula for black hole normal modes
}

\author{
M E Araújoł\|ף D, D Nicholsonł and B F Schutz§ \\ Department of Physics and Astronomy, University of Wales College of Cardiff, Cardiff CF1 \\ 3TH, UK
}

Received 8 February 1992

\begin{abstract}
We present a derivation of the Bohr-Sommerfeld formula used by Guinn et al to successfully calculate the complex eigenfrequencies for the low-lying quasi-normal modes of a Schwarzschild black hole. It is shown that this formula is valid even when the transition points under consideration come close together.
\end{abstract}

PACS numbers: $9760 \mathrm{~L}, 9880$

\section{Introduction}

In a recent paper, Guinn et al [1] (hereafter GWKs) have calculated complex eigenfrequencies of the quasi-normal modes of a Schwarzschild black hole, using an extension of the standard WKB technique into the complex frequency domain. At the lowest order of approximation, they used essentially the familiar Bohr-Sommerfeld (hereafter BS) quantization condition for complex points. This simplified method proved to be very precise for the low-lying modes, producing results in accordance with Leaver's [2], Nollert and Schmidt's [3] and Andersson's [4] numerical calculations, but it disagreed with these authors for the higherorder modes, in which the imaginary part of the frequency becomes large. GWKs used the BS formula without proof, so its failure to agree with other methods for higher modes casts doubt on its applicability to this problem or to the other methods. In this paper we resolve these doubts by proving that the BS formula is applicable to the lowest-order modes, and then demonstrate how it fails for the higher modes.

Our proof uses the phase-integral formalism of Fröman and Fröman\#, a generalization of the WKB method whose use for black-hole problems has been reviewed in [9]. The present paper can be read on its own, but readers wishing to follow all the details are referred to that review. Our results generalize the work of Thylwe [10] for Regge-Pole positions. An

† E-mail address: araujo@taff.cardiff.ac.uk

$\ddagger$ E-mail address: dxn@vl.astro.cf.ac.uk

§ E-mail address: schutz@cardiff.ac.uk

II Permanent address: Departamento de Matemática, Universidade de Brasinia, Caixa Postal 04670, 70.919-000 Brasilia DF, Brazil.

T Permanent E-mail address: marcelo@brunb.bitnet

\# The first-order phase-integral approximation is equivalent to the first-order wKB approximation [5]. Higher-order phase-integral approximations $[6,7]$ though not identical to the higher-order wKB approximations have in general the same accuracy. However, it is easier to establish enror bounds in the former (see [8]), 
improved, generalized Bohr-Sommerfeld formula has already been published [11], which corrects the major shortcomings of the GWKS formula for higher modes.

In section 2 we briefly review the phase-integral formalism using the ReggeWheeler [12] equation as a model problem for the application of the concepts as they are introduced. A more extensive review of this technique in the context of the black hole problem may be found in [9]. Section 3 gives a derivation of our result. In section 4 we present our conclusions and make suggestions for future work in this field.

\section{Review of the phase-integral method}

Consider the time-independent Schrödinger-like differential equation

$$
\frac{\mathrm{d}^{2} \Psi}{\mathrm{d} r^{2}}+R_{\ell}(r) \Psi=0
$$

where $r$ is a complex variable. In the case of a Schwarzschild black hole, we have [13]

$$
R_{\varepsilon}(r)=\frac{r^{2}}{(r-2)^{2}}\left(\sigma^{2}-V(r)-\frac{3}{r^{4}}+\frac{2}{r^{3}}\right)
$$

where $\sigma$ denotes the complex frequency, and where

$$
V_{\ell}(r)=\left(1-\frac{2}{r}\right)\left[\frac{\ell(\ell+1)}{r^{2}}-\frac{6}{r^{3}}\right]
$$

is the Regge-Wheeler (odd-parity) potential and $\ell$ is the spherical harmonic index. We have set $M=1$. One should obtain the same complex frequencies with the use of either the Regge-Wheeler potential or the Zerilli (even-parity) potential [14]. We use the former in the present work because it has a simpler functional form. We assume that the time factor of the solution of (1) is $\exp (-\mathrm{i} \sigma t)$.

The most general transformation of the dependent and independent variables that preserves the reduced form of (1) is given by [5]

$$
\begin{aligned}
& \Psi=[Q(r)]^{-1 / 2} \phi(r) \\
& \omega(r)=\int^{r} Q(\xi) \mathrm{d} \xi
\end{aligned}
$$

for arbitrary $Q$, and (1) thus transforms to

$$
\frac{\mathrm{d}^{2} \phi}{\mathrm{d} \omega^{2}}+(1+\epsilon) \phi=0
$$

where

$$
\epsilon=\frac{R-Q^{2}}{Q^{2}}+\frac{1}{16 Q^{6}}\left[5\left(\frac{\mathrm{d}}{\mathrm{d} r} Q^{2}\right)^{2}-4 Q^{2} \frac{\mathrm{d}^{2}}{\mathrm{~d} r^{2}} Q^{2}\right] .
$$

It is clear from the above that if we were to find a $Q$ that made $\epsilon=0$, the functions 


$$
\phi(r)=\mathrm{e}^{ \pm \mathrm{i} \omega(r)}
$$

would be linearly independent exact solutions of (6) and the general solution of (1) would be given by

$$
\Psi(r)=a_{1} f_{1}(r)+a_{2} f_{2}(r)
$$

where

$$
f_{1}(r)=Q^{-1 / 2}(r) \mathrm{e}^{\mathrm{i} \omega} \quad f_{2}(r)=Q^{-1 / 2}(r) \mathrm{e}^{-\mathrm{i} \omega}
$$

However, for potentials with complicated functional forms it is not possible in general to find $Q^{2}$. to make $\epsilon=0$ exactly. If one chooses $Q^{2}$ in such a way that $|\epsilon|$ is small compared with unity, then (8) still gives approximate solutions to the differential equation (1). The usual WKB approximation is obtained by taking $Q^{2}=R$, and is valid provided that $R(r)$ satisfies the usual restrictions that make (1) treatable by the WKB method [15].

Having chosen $Q^{2}$ in a satisfactory way, it is convenient to write

$$
\phi=a_{1}(\omega) \mathrm{e}^{\mathrm{i} \omega}+a_{2}(\omega) \mathrm{e}^{-\mathrm{i} \omega}
$$

to transform the second-order ordinary differential equation (6) into a coupled system of ordinary differential equations for the $a_{i}$. Imposing the condition that

$$
\frac{\mathrm{d} a_{1}}{\mathrm{~d} \omega} \mathrm{e}^{\mathrm{i} \omega}+\frac{\mathrm{d} a_{2}}{\mathrm{~d} \omega} \mathrm{e}^{-\mathrm{i} \omega}=0
$$

leads to

$$
\frac{\mathrm{d} a_{1}}{\mathrm{~d} \omega}=\frac{\mathrm{I}}{2} \mathrm{i} \epsilon\left[a_{1}+a_{2} \exp (-2 \mathrm{i} \omega)\right] \quad \frac{\mathrm{d} a_{2}}{\mathrm{~d} \omega}=-\frac{1}{2} \mathrm{i} \epsilon\left[a_{2}+a_{1} \exp (2 \mathrm{i} \omega)\right]
$$

The solution of the system (13) from initial data $a_{j}\left(\omega_{0}\right)$ at $\omega=\omega_{0}$ to any other $\omega$ may be given formally by

$$
a_{i}(\omega)=F_{i j}\left(\omega, \omega_{0}\right) a_{j}\left(\omega_{0}\right)
$$

Fröman and Fröman [5] give convergent series for the matrix $F_{i j}\left(\omega(r), \omega\left(r_{0}\right)\right)$ whose determinant is 1. They also give estimates of those series based on the assumptions that $r$ and $r_{0}\left(\omega\right.$ and $\left.\omega_{0}\right)$ can be connected by a path $\Lambda$ in the complex $r$-plane ( $\omega$-plane) along which $\left|e^{\mathrm{i} \omega(r)}\right|$ increases monotonically from $r_{0}$ to $r$ and an error bound defined as

$$
\mu\left(r, r_{0}\right)=\int_{\Lambda}|\epsilon(\xi) Q(\xi) \mathrm{d} \xi|
$$

is much smaller than 1 .

In the case of Schwarzschild black holes, $R(r)$ as given by (2) and (3) is singular both at the origin and at the horizon. It is the latter singularity that should be cause for concern, since we must be able to continue a solution valid at the horizon, where we place a boundary 
condition of purely ingoing waves, into other regions of the complex plane. In order to satisfy this requirement we use our freedom in choosing $Q^{2}$ and follow Fröman and Fröman (see chapter 11 in [5]) to write

$$
Q^{2}(r)=R(r)-\frac{1}{4(r-2)^{2}}
$$

Given that $R$ has a second-order pole at the horizon this choice of $Q^{2}$ makes the integrand of the $\mu$-integral (15) analytic at the horizon, which is a necessary condition for having a finite error bound when a solution is continued from the horizon to other regions of the complex plane.

We have investigated the behaviour of $|\epsilon Q|$ in the neighbourhood of the horizon, using the eigenfrequency of some of the low-lying modes, and found that the error bound seems to be high for some of the possible paths along which one would continue the solution. Indeed Guinn $e$ al by taking $Q^{2}=R$, the usual WKB approximation, have a divergent $\mu$ integral. This is a striking and unexpected feature since, as mentioned in the introduction, the Bs formula gives accurate results for the low-lying modes. It is therefore clear that error estimates within the phase-integral formalism need further consideration. This problem is now being investigated.

Note that to obtain $Q$ and $\omega$ one has to calculate the square root of (16). We then assume that the complex $r$-plane is cut and a multiple-sheet Riemann surface introduced to make both $Q$ and $\omega$ single-valued functions (see figure 1).

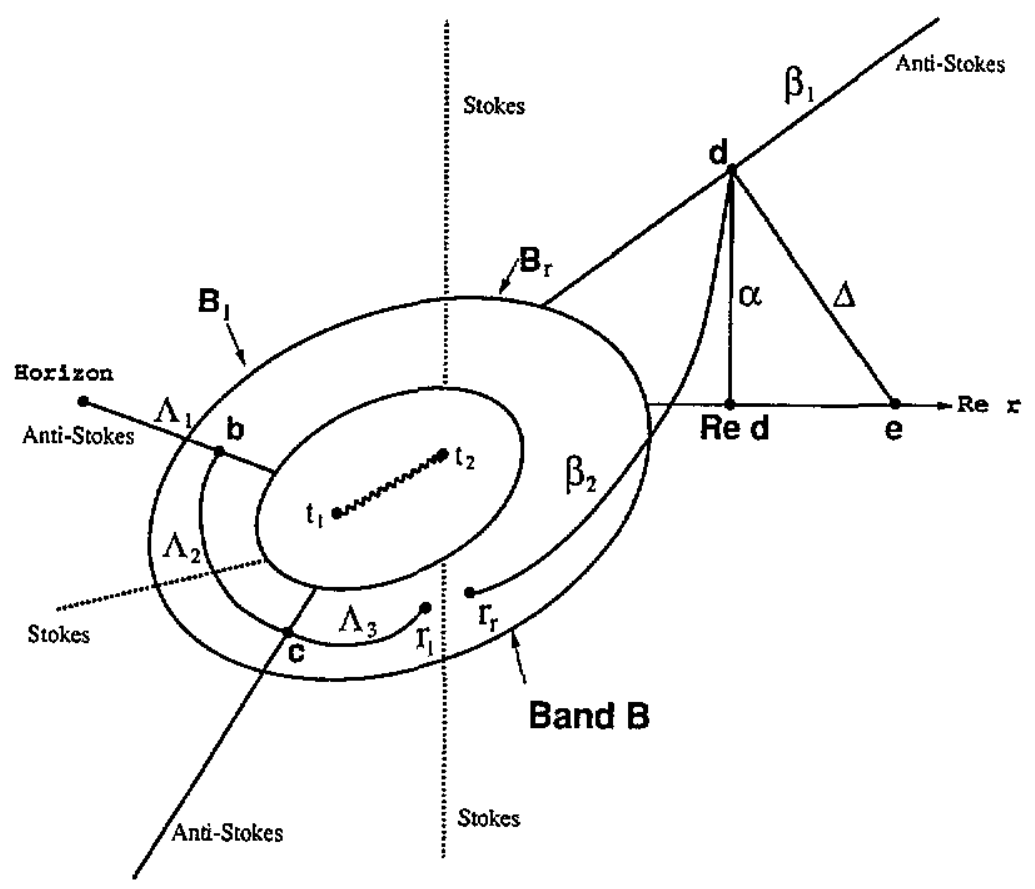

Figure 1. Schematic representation of the complex $r$-plane showing the pattern of Stokes and anti-Stokes lines characteristic of a two-transition-point problem and a band $B$ divided in two regions $B_{1}$ and $B_{\mathrm{r}}$ by Stokes lines. The wavy line denotes a cut. 
From (2), (3) and (16) we obtain

$$
\begin{aligned}
& \lim _{r \rightarrow \infty} Q^{2}(r)=\sigma^{2} \\
& \lim _{r \rightarrow+2}(r-2)^{2} Q^{2}(r)=4 \sigma^{2} .
\end{aligned}
$$

We choose the phase of $Q(r)$ such that

$$
\begin{aligned}
& \lim _{r \rightarrow \infty} Q(r)=\sigma \\
& \lim _{r \rightarrow+2}(r-2) Q(r)=-2 \sigma .
\end{aligned}
$$

It has been shown by Regge and Wheeler [12] that the energy integral and the boundary conditions of purely ingoing waves at the horizon and purely outgoing waves at infinity lead to damped stable quasi-normal modes. Therefore, with our choice of time factor we must have $\operatorname{Im} \sigma<0$.

An important concept in the analysis of a differential equation in the complex coordinate plane is the role of the so-called Stokes and anti-Stokes lines. If $\epsilon=0$, these are curves on which $\omega$, or equivalently $Q \mathrm{~d} r$, is pure imaginary or real respectively. The equations $\operatorname{Re}(Q \mathrm{~d} r)=0(\mathrm{Im}(q \mathrm{~d} r)=0)$ are equivalent to a two-dimensional autonomous system of differential equations. The zeros of $Q$ are the fixed points of the system. Since in general one is not able to solve $\epsilon=0$ exactly, one has to work with the so-called semi-classical Stokes (anti-Stokes) lines, i.e. curves satisfying the condition $Q \mathrm{~d} r$ pure imaginary (real) for some function $Q$ for which $|\epsilon|$ is small compared with unity. From this definition, it is clear that the solutions ( 8 ) behave as travelling waves along anti-Stokes lines and that their ratio remains the same size all along the line.

The introduction of boundary conditions of outgoing waves at infinity and waves falling across the horizon on the real coordinate axis leads to well known numerical difficulties [14], since the desired solutions of (1) are exponentially increasing as the horizon and infinity are approached. Therefore, in order to uniquely define a quasi-normal mode, we have to single out an exponentially decreasing term from the required increasing one. This is a delicate problem, since the decreasing term will eventually become smaller than the error introduced in the increasing one.

This problem can be avoided by placing the boundary conditions on anti-Stokes lines than run towards the horizon and infinity. It is shown in appendix B that the boundary condition of outgoing waves placed on the anti-Stokes lines emerging from the region where the two transition points lie towards infinity, leads to the correct boundary conditions at real infinity.

The detailed description of the process leading to the calculation of the Stokes and anti-Stokes lines is given in appendix $\mathrm{A}$. The computer programs for performing these calculations can be obtained from any of the authors upon request.

\section{Derivation of the Bohr-Sommerfeld formula}

By virtue of our choice of cuts in the complex plane and phase for $Q(r),(19)$ and (20), we have that $f_{1}(r)$ in (10) represents outgoing waves as $r \mapsto \infty$ and ingoing waves as $r \mapsto+2$. 
Since there are no waves coming out from the black hole, near the horizon we must have that the function $\Psi$ takes the asymptotic form

$$
\Psi \underset{r \rightarrow+2}{\sim} a_{1} f_{1}(r)
$$

i.e. $a_{2} \equiv a_{2}(+2)=0, a_{1} \equiv a_{1}(+2)$ is an undetermined nomalization factor. In analogy with the demonstration given in the appendix of [9] it is possible to show that our choice of $Q^{2}$ and the boundary condition for quasi-normal modes at the horizon allow us to write

$$
a_{1}(r)=F_{11}(r,+2) a_{1}(+2) \quad a_{2}(r)=F_{21}(r,+2) a_{1}(+2) .
$$

Using (14), (21) and (22) we obtain that at an arbitrary point $r$ in the complex coordinate plane $\Psi$ can be written as

$$
\Psi(r)=\left[F_{11}(r,+2) f_{1}(r)+F_{21}(r,+2) f_{2}(r)\right] a_{1}(+2) .
$$

In particular for $r \mapsto \infty$ we have

$$
\Psi \underset{r \rightarrow \infty}{=}\left[F_{11}(\infty,+2) f_{1}(r)+F_{21}(\infty,+2) f_{2}(r)\right] a_{1}(+2)
$$

The boundary condition of purely outgoing waves at infinity means that we must have $F_{21}(\infty,+2)=0$ and in the limit for $r \mapsto \infty$ the function $\Psi$ is

$$
\Psi \underset{r \rightarrow \infty}{\sim} b_{1} f_{l}(r) \quad b_{1}=a_{1}(+2) F_{11}(\infty,+2) .
$$

Following Thylwe [10], our next step is to show that $\Psi$ has approximately the same functional form as in (21) and (25) in a certain band in the complex $r$-plane which surrounds the two transition points $t_{1}$ and $t_{2}$ under consideration lying far away from them and from the other transition points (figure 1). If these two transition points, which may be close to each other, are well separated from the other transition points, one has a pattern of Stokes and anti-Stokes lines emerging from them that is characteristic of a two-transition-point problem consisting of first-order zeros [16].

Although for the present analysis we do not need to know the precise configuration of Stokes and anti-Stokes lines, we need to use the fact that there is an anti-Stokes line running from the region containing the transition points to the horizon and another anti-Stokes line running from the same region to infinity such that both $\operatorname{Re} r \mapsto \infty$ and $\operatorname{Im} r \mapsto \infty$.

We start by deriving an expression for $\Psi$ at a point $r$ in the left side of the band $B$, denoted $B_{1}$. For that purpose we consider a path $\Lambda$ divided in three subpaths $\Lambda_{1}, \Lambda_{2}$ and $\Lambda_{3}$. The subpath $\Lambda_{1}$ runs along an anti-Stokes lines from the horizon to a point $b . \Lambda_{2}$ connects $b$ to a point $c$ on the neighbouring anti-Stokes line and $\Lambda_{3}$ links $c$ to $r_{1}$ in such a way that $|\exp (i \omega)|$ is monotonically increasing (see figure 1 ).

Now, in order to continue the solution from the horizon to $r_{!}$we have to calculate the $F$-matrix connecting them. It follows from (14) that $F_{i j}\left(+2, r_{1}\right)$ can be written as the product of three matrices, namely,

$$
F_{i j}\left(+2, r_{1}\right)=F_{i k}(+2, b) F_{k l}(b, c) F_{l j}\left(c, r_{1}\right) .
$$

Since the subpath $\Lambda_{1}$ connects two points on an anti-Stokes line we can use the basic estimates given in [9] to obtain

$$
F_{i j}(+2, b)=\left(\begin{array}{ll}
1 & 0 \\
0 & 1
\end{array}\right)+O(\mu)
$$


The subpath $\Lambda_{2}$ connects two points on neigbouring anti-Stokes lines along which $|\exp (i \omega)|$ has a single minimum. The $F$-matrix connecting $b$ and $c$ is then given by expression (3.7) in [16], namely

$$
F_{i j}(b, c)=\left(\begin{array}{ll}
1 & ? \\
0 & 1
\end{array}\right)+\mathrm{O}(\mu)
$$

where '?' denotes that $F_{12}$ is unknown and may be large.

From the characteristics of the subpath $\Lambda_{3}$ described above we find, using the basic estimates of chapter 4 in [5], that

$$
F_{i j}\left(r_{1}, c\right)=\left(\begin{array}{cc}
1+\mathrm{O}(\mu) & \mathrm{O}(\mu) \\
\mathrm{O}(\mu)\left|\mathrm{e}^{2 \mathrm{i} \omega\left(r_{1}\right)}\right| & ?
\end{array}\right) .
$$

Now, using the inverse of (26) and (21) it follows that

$$
\Psi\left(r_{1}\right)=a_{1}(+2) f_{1}\left(r_{1}\right)\left[1+o\left(\mu_{1}\right)\right] .
$$

We now wish to obtain an expression for $\Psi$ at a point $r_{\mathrm{r}}$ in the right side of the band $B$, denoted $B_{\mathrm{r}}$. We consider a path $\beta$ divided into two subpaths $\beta_{1}$ and $\beta_{2}$. The subpath $\beta_{1}$ runs along the anti-Stokes line from infinity to $d$ and subpath $\beta_{2}$ connects $d$ to $r_{\mathrm{r}}$ in such a way that along $\beta_{2}|\exp (i \omega)|$ is monotonically increasing (figure 1).

Again, in order to continue the solution from infinity to $r_{\mathrm{r}}$ we have to calculate $F_{i j}\left(\infty, r_{\mathrm{r}}\right)$ which can be expressed as the product of two matrices,

$$
F_{i j}\left(\infty, r_{\mathrm{r}}\right)=F_{i k}(\infty, d) F_{k j}\left(d, r_{\mathrm{r}}\right)
$$

The subpath $\beta_{1}$ connects points on an anti-Stokes lines. Thus,

$$
F_{i k}(\infty, d)=\left(\begin{array}{ll}
1 & 0 \\
0 & 1
\end{array}\right)+O(\mu)
$$

Using the basic estimates of chapter 4 in [5] and the characteristics of subpath $\beta_{2}$ described above we obtain

$$
F_{i j}\left(r_{\mathrm{r}}, d\right)=\left(\begin{array}{cc}
1+\mathrm{O}(\mu) & \mathrm{O}(\mu) \\
\mathrm{O}(\mu)\left|\mathrm{e}^{2 \mathrm{i} \omega\left(r_{\mathrm{s}}\right)}\right| & ?
\end{array}\right) .
$$

Finally, using (25), (31), (32) and (33) we find that

$$
\Psi\left(r_{\mathrm{r}}\right)=b_{\mathrm{l}} f_{\mathrm{l}}\left(r_{\mathrm{r}}\right)\left[1+\mathrm{O}\left(\mu_{\mathrm{r}}\right)\right] \text {. }
$$

We have mentioned in the previous section, that the boundary condition of outgoing waves at infinity is placed on the anti-Stokes line that runs from the region containing the transition points to infinity. It must then be demonstrated that this procedure leads to the correct boundary condition at real infinity. This is equivalent to showing that, in the limit for $d \mapsto \infty$ along $\beta_{1}$, there exists a path running from $d$ to a point in the real axis such that the $F$-matrix connecting them is the identity matrix. In appendix $\mathrm{C}$ we demonstrate the existence of such a path and comment that it is not unique. 
Since the exact solution $\Psi$ is continuous in the common boundary of the regions $B_{1}$ and $B_{\mathrm{r}}$, it is reasonable to expect that if $\Psi\left(r_{\mathrm{l}}\right)$ and $\Psi\left(r_{\mathrm{r}}\right)$ are to be good approximations to the exact solution, they must both be valid in that region. Therefore, it follows from equating (30) and (34) that

$$
b_{1}=a_{1}(+2)[1+\mathrm{O}(\mu)] \text {. }
$$

We have thus established that in the band $B$

$$
\Psi(r)=a_{1} f_{1}(r)[1+\mathrm{O}(\mu)] \quad \frac{\mathrm{d} \Psi}{\mathrm{d} r}(r)=a_{1} \frac{\mathrm{d} f_{1}}{\mathrm{~d} r}(r)[1+\mathrm{O}(\mu)] .
$$

The exact solution $\Psi(r)$ is an analytic function in the band $B$ and in the region surrounded by B except possibly for poles. It then follows [17] that the function $\Psi^{\prime}(r) / \Psi(r)$ is an analytic function of $r$ in the region with simple poles at the zeros and poles of $\Psi$. The residue of a pole of order $m$ is $-m$ and at a zero of order $n$ is $n$. It is reasonable to expect that in the present case both $m$ and $n$ are integers. Therefore, using Cauchy's residue theorem we obtain

$$
\oint_{C} \frac{\Psi^{\prime}(r)}{\Psi(r)} \mathrm{d} r=2 \pi \mathrm{i} N
$$

where $N=\sum n-\sum m$ and we choose $C$ to be a closed contour in the band $B$ encircling the zeros and poles of $\Psi$ in the positive sense. Assuming that expressions (36) are good approximations to the exact solution, we can approximate the integrand in (37) using them, and thus obtain

$$
\frac{\Psi^{\prime}(r)}{\Psi(r)}=-\frac{\mathrm{d}}{\mathrm{d} r} \ln Q^{1 / 2}+\mathrm{i} Q+\mathrm{O}(\mu) .
$$

Finally, using (38) in (37) we obtain the Bohr-Sommerfeld formula

$$
\frac{1}{2} \oint_{C} Q(r) \mathrm{d} r=\left(N+\frac{1}{2}\right) \pi+O(\mu) .
$$

We note that the integration procedure does not introduce further errors since the integrand is a slowly varying function on the band $B$.

\section{Conclusions}

We have shown that the BS formula can be obtained by a straightforward application of the phase-integral formalism to the Regge-Wheeler equation, even when the two transition points under consideration cannot be considered well separated from each other. They must, however, be well isolated from the other transition points.

We have demonstrated that the boundary conditions for outgoing waves at infinity, placed on the anti-Stokes lines emerging from the region where the two transition points lie towards infinity (figure 1), leads to the correct boundary conditions at real infinity. This is a very important feature, since we now have a clear way of avoiding the numerical difficulties of tracking an exponentially decreasing term from the required increasing one [14]. 
The BS formula is very accurate for the determination of the low-lying quasi-normal modes because the influence of the other transition points on the pattern of Stokes and antiStokes on the band $B$ is negligible. For higher values of the overtone index $n$ this infuence becomes significant and third transition point must be taken into account (see [11]).

It was pointed out that the error bound, the $\mu$-integral, seems to be high for some of the possible paths along which one would continue the solution despite the accuracy of the BS formula for the calculation of the eigenfrequencies for the low-lying quasi-normal modes. We are at present carefully considering this question which is intrinsically connected with the more general topic of estimates of error bounds within the phase-integral formalism.

\section{Acknowledgments}

DN is in receipt of an award from the SERC. MEA wishes to thank CAPES and the University of Brasilia for finantial support and the Department of Physics of the University of Wales College of Cardiff for its hospitality. We are all indebted to Dr Nils Andersson for useful discussions during the preparation of this manuscript.

\section{Appendix $\mathbf{A} \dagger$}

In what follows, we will describe in detail the determination of the autonomous system of differential equations that one obtains from the equation for (semiclassical) anti-Stokes lines. Analogous steps can be followed for the equation for (semiclassical) Stokes lines.

Semiclassical anti-Stokes lines are the integral curves of the system of ordinary differential equations obtained from the complex equation

$$
\operatorname{Im}\left(Q^{1 / 2} \mathrm{~d} z\right)=0
$$

where

$$
Q=R(z)-\frac{1}{4(z-2)^{2}}
$$

In the region close to a zero of $Q$, we then factorize it as

$$
Q=\left(z-z_{t}\right) f(z)
$$

where $z_{t}$ are the coordinates of the zero of $Q$. We then write,

$$
\begin{aligned}
& z-z_{t}=r \mathrm{e}^{\mathrm{i} \theta} \\
& \mathrm{d} z=(\mathrm{d} r+\mathrm{i} r \mathrm{~d} \theta) \mathrm{e}^{\mathrm{i} \theta} \\
& \mathrm{d} r+\mathrm{i} r \mathrm{~d} \theta=\mathrm{d} s \mathrm{e}^{\mathrm{i} \phi} \\
& f(z)=|f(z)| \mathrm{e}^{\mathrm{i} \alpha}
\end{aligned}
$$

In this appendix $r$ is a real variable and $r=0$ is the coordinate of the zero of $Q$ under consideration. 
from which it follows that

$$
\begin{aligned}
& \mathrm{d} r=\mathrm{d} s \cos \phi \\
& r \mathrm{~d} \theta=\mathrm{d} s \sin \phi
\end{aligned}
$$

Then

$$
Q^{1 / 2} \mathrm{~d} z=(|f(z)| r)^{1 / 2} \exp (3 \theta / 2+\alpha / 2+\phi) \mathrm{d} s .
$$

The equation (A1) is then equivalent to

$$
\frac{3 \theta}{2}+\frac{\alpha}{2}+\phi=n \pi
$$

Finally, we substitute (11) into (A8) and (A9) to obtain

$$
\begin{aligned}
& \frac{\mathrm{d} r}{\mathrm{~d} s}=(-1)^{n} \cos \left(\frac{3 \theta+\alpha}{2}\right) \\
& \frac{\mathrm{d} \theta}{\mathrm{d} s}=\frac{(-1)^{n+1}}{r} \sin \left(\frac{3 \theta+\alpha}{2}\right) .
\end{aligned}
$$

Equations (A12) and (A13) are the ones we use to obtain the anti-Stokes lines. The three lines emerging with angles of $120^{\circ}$ between them are a result of setting $3 \theta+\alpha / 2=n \pi$ in (A13) to avoid infinities at $r=0$.

\section{Appendix B}

We intend to show that in the limit for $d \mapsto \infty$, along the anti-Stokes line where it lies, there exists a path running from $d$ to a point in the real axis such that the $F$-matrix connecting them is the identity matrix. This result follows from the basic estimates of chapter 4 in [5], provided that along such a path $|\exp (i \omega)|$ is monotonically increasing and that the error bound, the $\mu$-integral, tends to zero.

Consider a path $\alpha$ being a straight line orthogonal to the real axis connecting $d$ to $\operatorname{Re} d$ (figure 1). We start by showing that, for $d \mapsto \infty$ along $\beta_{1},|\exp (\mathrm{i} \omega)|$ is monotonically increasing along $\alpha$.

From the definiton (5) it follows that

$$
\omega(\operatorname{Re} d)=\omega(d)+\int_{\alpha} Q \mathrm{~d} r .
$$

Using (19), we obtain that far away from the region $B$

$$
\int_{\alpha} Q \mathrm{~d} r=\int_{y^{\prime}}^{0} Q \mathrm{~d} y=-\mathrm{i} y^{\prime} \sigma
$$

where we have introduced the notation $r=x+\mathrm{i} y$ and $d=x^{\prime}+\mathrm{i} y^{\prime}$. From (B1) and (B2) we have that

$$
\left|\exp \left[i \omega\left(x^{\prime}\right)\right]\right|=\left|\exp \left(y^{\prime} \operatorname{Re} \sigma\right)\right|
$$


It follows from $\operatorname{Re} \sigma>0$ and $y^{\prime} \mapsto \infty$ that $|\exp (\mathrm{i} \omega)|$ is monotonically increasing along $\alpha$.

We now show that for $d \mapsto \infty$ along $\beta_{1}$ the $\mu$-integral tends to zero along $\alpha$. From (7), (15) and (19) we have that

$$
\lim _{\substack{d \rightarrow \infty \\ \text { along } \beta_{1}}} \mu=\frac{1}{4|\sigma|} \lim _{\substack{y^{\prime} \rightarrow \infty \\ x^{\prime} \rightarrow \infty}} \int_{y^{\prime}}^{0} \frac{d y}{\left[\left(x^{\prime}-2\right)^{2}+y^{2}\right]}=0
$$

We point out that the path $\alpha$ is a 'good path' in the sense that it satisfies the necessary assumptions that allow us to use the basic estimates in [5] and is obviously simple to describe. However, it would have been more elegant to use a Stokes line orthogonal to the anti-Stokes line on which $d$ lies to connect it to a point in the real axis.

Far away from the transition points, both the Stokes and anti-Stokes lines under consideration tend to be straight lines. It follows that along such Stokes line ( $\Delta$ in figure 1 ) the integral in (B2) would be replaced by

$$
\mathrm{i} \frac{|\sigma|^{2}}{\operatorname{Im} \sigma} \int_{x^{\prime}}^{x^{\prime}-(\operatorname{Im} \sigma / \operatorname{Re} \sigma) y^{\prime}} \mathrm{d} x=-\mathrm{i}|\sigma|^{2} \frac{y^{\prime}}{\operatorname{Re} \sigma}
$$

and in this case (B3) would be replaced by

$$
\left|\exp \left[i \omega\left(x^{\prime}\right)\right]\right|=\left.\left|\frac{y^{\prime}}{\operatorname{Re} \sigma} \exp \right| \sigma\right|^{2} \mid
$$

and using the same argument as before it follows that $|\exp (i \omega)|$ is monotonically increasing along this path.

We also have that, for $d \mapsto \infty$, the $\mu$-integral along $\Delta$ is given by

$$
\begin{aligned}
& \lim _{\substack{d \rightarrow \infty \\
\text { along } \beta_{1}}} \mu=\lim _{\substack{y^{\prime} \rightarrow \infty \\
x^{\prime} \rightarrow \infty}} \frac{1}{4 \operatorname{Re} \sigma} \int_{y^{\prime}}^{0} \frac{1}{A y^{2}+B y+C} \mathrm{~d} y=0 \\
& A=1+\left(\frac{\operatorname{Im} \sigma}{\operatorname{Re} \sigma}\right)^{2} \\
& B=2 \frac{\operatorname{Im} \sigma}{\operatorname{Re} \sigma}\left[\left(x^{\prime}-2\right)-y^{\prime} \frac{\operatorname{Im} \sigma}{\operatorname{Re} \sigma}\right] \\
& C=\left[\left(x^{\prime}-2\right)-y^{\prime} \frac{\operatorname{Im} \sigma}{\operatorname{Re} \sigma}\right]^{2} .
\end{aligned}
$$

Finally, it follows from the basic estimates given in [9] that

$$
\lim _{\substack{d \rightarrow \infty \\
\text { along } \beta_{1}}} F_{i j}(d, \operatorname{Re} d)=\left(\begin{array}{ll}
1 & 0 \\
0 & 1
\end{array}\right) \quad \lim _{\substack{d \rightarrow \infty \\
\text { along } \beta_{1}}} F_{i j}(d, e)=\left(\begin{array}{ll}
1 & 0 \\
0 & 1
\end{array}\right)
$$




\section{References}

[1] Guinn J W, Will C M, Kojima Y and Schutz B F 1990 High-overtone normal modes of Schwarzschild black holes Class. Quantum Grav. 7 LA7-L53

[2] Leaver E W 1985 An analytic representation for the quasi-normal modes of Kerr black holes Proc. R. Soc. A $402285-98$

[3] Nollert H-P and Schmidt B G 1992 Quasinormal modes of Schwarzschild black holes: Defined and calculated via Laplace transformation Phys. Rev. D 45 2617-27

[4] Andersson N 1992 A numerically accurate investigation of black hole normal modes Proc. R. Soc, A 439 47-58

[5] Fröman P O and Fröman N 1965 JwKB Approximation, Contributions to the Theory (Amsterdam: NorthHolland)

[6] Froman N 1966 Outline of a general theory for higher-order approximations of the swKB-type Ark. Fys. 32 $541-8$

[7] Fröman N and Froman P O 1974 A direct method for modifying certain phase-integral approximations of arbitrary order Ann. Phys. 83 103-7

[8] Fröman N and Fröman P O 1992 Phase-integral approximation of arbitrary order generated from an unspecified base function Forty More Years of Ramifications: Spectral Asymptotics and its Applications (Discourses in Mathematics and its Applications no I) ed S A Fulling and F J Narcowich (Texas A and $M$ University, Department of Mathematics)

[9] Andersson N. Araujo M E and Schutz B F 1993 The phase-integral method and black-hole normal modes Class. Quantum Grav. $10735-55$

[10] Thylwe K-E 1985 Alternative Derivation of a Regge-Pole condition of the Bohr-Sommerfeld type J. Phys. A: Math. Gen. 18 3445-53

[11] Andersson N, Araujo M E and Schutz B F 1993 Generalized Bohr-Sommerfeld formula for Schwarzschild black hole normal modes Class. Quantum Grav. $10757-65$

[12] Regge T and Wheeler J A 1957 Stability of a Schwarzschild singularity Phys. Rev. 108 1063-9

[13] Fróman N, Fröman P O, Andersson N and and Höback A 1992 Black-hole normal modes: phase-integral treatment Phys. Rev. D 45 2609-16

[14] Chandrasekhar S and Detweiler S 1975 The quasi-normal modes of the Schwarzschild black hole Proc. $R$. Soc. A 344 441-52

[15] Mathews J and Walker R L 1970 Mathematical Methods of Physics 2nd edn (Reading, MA: Addison-Wesley)

[16] Fröman N, Fröman P O and Lundborg B 1988 The Stokes constants for a cluster of transition points Math. Proc. Camb. Phil. Soc. 104 153-79

[17] Jeffreys H and Jeffreys B S 1946 Methods of Mathematical Physics (Cambridge: Cambridge University Press) 